Website: http://revistas.lamolina.edu.pe/index.php/acu/index

(C) Universidad Nacional Agraria La Molina, Lima - Perú

\title{
Evaluación del tamaño de lote en la crianza comercial de cuyes (Cavia porcellus) en la etapa de crecimiento
}

\author{
Evaluation of the size of lot in the commercial breeding of guinea pigs (Cavia porcellus) in the \\ stage of growth
}

Erik Roter ${ }^{1}$, Wilder Trejo ${ }^{2 *}$ y Gloria Palacios ${ }^{3}$

* Autor de correspondencia

\begin{abstract}
Resumen
Con el objetivo de evaluar el efecto del tamaño del lote sobre la performance de los animales y retribución económica. Se evaluó lotes de 10, 30 y 50 cuyes machos destetados del tipo I (14 \pm 3 días $)$, criados en poza y usando un área libre de $0.0781 \mathrm{~m}^{2}$ por animal, distribuidos en bloques de acuerdo al peso de destete. No se encontraron diferencias significativas en el consumo de materia seca, número de animales retrasados en el crecimiento, número de carcasas dañadas, ni en el rendimiento de carcasa. Sin embargo, la cantidad de animales instalados en la poza afectó, con diferencia estadística significativa ( $\mathrm{p} \leq 0.05)$, la ganancia de peso y conversión alimenticia, mostrando que existe una relación directa entre el tamaño de lote y la respuesta productiva del animal. El lote de 10 animales obtuvo los mejores resultados en ganancia de peso y conversión alimenticia. Por otro lado, la retribución económica evaluada para unidad de carcasa mostró respuestas similares entre todos los tamaños de lote y para kilogramo de carne fue mejor la crianza en lotes de 10 animales.
\end{abstract}

Palabras clave: área de crianza; cuy; parámetros productivos; tamaño de lote.

\begin{abstract}
With objective to evaluate effects of the size of lot on the performance of the animals and economic retribution. Evaluated lots of 10, 30 and 50 guinea pigs weaned male from the type I (14 \pm 3 days), reared in barnyard and using a free area of $0.0781 \mathrm{~m}^{2}$ per animal, distributed in blocks according to the weight of weaning. No significant differences were found in the dry matter intake, number of animals in the retarded growth, number of damage carcasses or carcass yield. However, the amount of animals in the barnyard had affected, with statistically significant difference $(\mathrm{p} \leq 0.05)$, the weight gain and feed conversion, showing that there is a direct relationship between the lot size and the productive response of the animal. The lot of 10 animals obtained the best results in weight gain and feed conversion. On the other hand, the economic retribution evaluated for carcass unit showed similar responses between all sizes of lots and for kilogram of meat was better breeding in groups of 10 animals.
\end{abstract}

Keywords: breeding area; guinea pig; productive parameters; size of lots.

\section{Introducción}

El cuy es una especie criada y consumida desde tiempos inmemoriales, evidenciándose que fue muy importante para la población del Perú antiguo. Hoy en día, si bien se le sigue criando de forma tradicional familiar, se ha impulsado la crianza comercial con poblaciones que superan las 1000 madres y que, sin embargo, se vienen manejando en lotes pequeños entre 6 a 10 animales por poza (Cayotopa, 1986), haciendo el trabajo tedioso y usando mayor mano de obra para esta explotación.

Por otro lado, el entorno o medio ambiente que rodea a los animales, influye en su comportamiento productivo (Carpenter, 1995). Es así que dentro de los factores ambientales, donde se encuentra el espacio vital, juega un rol importante los aspectos de estrés, produciendo respuesta de ansiedad o miedo que determinan reacciones fisiológicas, metabólicas y conductuales (Wellock et al., 2004). Todas las respuestas a factores estresantes están coordinadas por el sistema nervioso y endocrino; sin embargo, son varios los sistemas involucrados, tales como digestivo, inmunológico y reproductivo. Aguila (1999) sugiere que de acuerdo al tipo, intensidad y frecuencia del factor estresante, experiencia, genética, sexo y estado fisiológico del animal, la respuesta biológica global puede variar en tres formas: Evasión (física o mental), donde el animal cambia de comportamiento para evadir el factor estresante y si no hay forma de evadir aparecen conductas que no guardan relación con la situación; emergencia, 
cuando el factor estresante aparece súbitamente y con alta intensidad, la respuesta es dada por el sistema nervioso autónomo, médula y corteza adrenal observándose comportamientos de huida o pelea y resistencia. Cuando el factor estresante es crónico hay necesidad de energía a largo plazo, la producción de cortisol conduce a una alta demanda de nutrientes y tiene efecto inmunodepresor, por lo que el animal presenta retraso en el crecimiento, falla reproductiva y mayor incidencia de enfermedades (Wellock et al., 2004).

El espacio vital reducido genera incomodidad para alimentarse, desplazarse, descansar, etc., afectando niveles productivos y reproductivos. Los estudios realizados para determinar requerimientos de espacio vital en cuyes se remonta a la década de los setenta con áreas de 0.06 a $0.14 \mathrm{~m}^{2} /$ animal en recría en poza (Humala, 1971; Agustín, 1973); sin embargo, las prácticas de selección y mejoramiento genético han hecho posible que, en la actualidad, los cuyes son de mayor tamaño y peso, lo que hace lógico deducir que los requerimientos de espacio vital han variado. No obstante, Valverde (2006) obtuvo el mejor mérito económico con un área de $0.0781 \mathrm{~m}^{2} /$ cuy macho en recría. Por otro lado, Montesinos (1972) declara que una excesiva concentración de animales siempre trae como consecuencia peleas, sobre todo en machos; dando como resultado disminución en la ganancia de peso y baja conversión alimenticia. Asimismo, como resultado de las peleas se ocasionan lesiones en la carcasa y mayor incidencia de enfermedades de la piel.

El estudio tuvo como objetivo evaluar lotes de 10,30 y 50 cuyes machos por poza, usando un área libre de 0.0781 $\mathrm{m}^{2}$ por animal en la etapa de crecimiento, distribuidos en tres bloques de acuerdo al peso vivo inicial, a los que se evaluó sus parámetros productivos medido a través de la ganancia de peso, consumo de alimento, conversión alimenticia, rendimiento de carcasa, porcentaje de animales retrasados por lote (peso), porcentaje de carcasas dañadas y retribución económica.

\section{Materiales y métodos}

El presente estudio se llevó a cabo en las instalaciones de la Granja de Cuyes de Cieneguilla del Programa de Investigación y Proyección Social en Carnes de la Universidad Nacional Agraria La Molina, Lima, Perú, durante los meses de agosto a octubre del 2009.

Se utilizaron un total de 270 cuyes machos destetados (14 \pm 3 días) del tipo I se mantuvieron en producción hasta los 71 días de edad y fueron agrupados de acuerdo al peso vivo y dentro de cada grupo distribuidos completamente al azar en tres tratamientos con diferente tamaño de lotes y cada tratamiento con tres repeticiones; con un área total de $0.09 \mathrm{~m}^{2}$ por animal, de la cual $0.0781 \mathrm{~m}^{2}$ es libre y $0.0119 \mathrm{~m}^{2}$ como área de comedero, tal como se detalla en el Tabla 1. Se utilizó el mismo manejo para todos los cuyes, la crianza fue en pozas de concreto armado y la alimentación mixta, un alimento balanceado (peletizado) que fue suministrado Ad libitum, según las recomendaciones del NRC y rastrojo de brócoli de manera restringida (20\% del peso vivo) como forraje verde (Rivas, 1995). No se usó agua de bebida.

Tabla 1. Distribución de los animales en los diferentes tratamientos evaluados

\begin{tabular}{ccccc}
\hline \multirow{2}{*}{ Tratamiento } & \multirow{2}{*}{ Lote } & $\begin{array}{c}\text { Área total/ } \\
\text { Lote }\end{array}$ & $\begin{array}{c}\text { Área } \\
\text { ocupada por Pozas (n) } \\
\text { comedero }\end{array}$ & \\
\hline T 1 & 10 animales/poza & $0,9 \mathrm{~m}^{2}$ & $0,119 \mathrm{~m}^{2}$ & 3 \\
T 2 & 30 animales/poza & $2,7 \mathrm{~m}^{2}$ & $0,357 \mathrm{~m}^{2}$ & 3 \\
T 3 & 50 animales/poza & $4,5 \mathrm{~m}^{2}$ & $0,595 \mathrm{~m}^{2}$ & 3 \\
\hline
\end{tabular}

Los parámetros a evaluar durante el tiempo de estudio fueron: ganancia de peso, para lo cual fueron pesados semanalmente a la misma hora antes del suministro de alimentos; consumo de alimento balanceado que fue determinado semanalmente, pesándose cada siete días el alimento no consumido respecto al total ofrecido. El consumo de forraje verde fue calculado diariamente. c) La conversión alimenticia (C.A), se calculó en base al consumo de materia seca (concentrado + forraje) que requiere el animal para producir un kilogramo de peso vivo. d) Animales retrasados, se calculó mediante un registro de pesos semanales de cada animal debidamente identificados (se sacó un promedio de peso), los animales que están por debajo del 10\% del promedio se consideró como animales que acusaban retraso en su crecimiento. e) Rendimiento de carcasa, fue evaluado en 27 cuyes elegidos al azar, se dejaron en ayuno de $12 \mathrm{~h}$ antes del sacrificio; la carcasa estuvo conformada por piel, cabeza, patas y vísceras rojas (corazón, pulmones, hígado y riñones). f) Porcentaje de carcasas dañadas, fue evaluado por las heridas que presentan en cualquier parte del cuerpo producto de peleas entre los animales perjudicando la calidad de la misma. Esto se evaluó al tacto al momento de pesar a los animales (animal vivo); así como también visualmente al momento del beneficio. g) La retribución económica se evaluó en base al costo de alimentación por animal.

Se utilizó un diseño de bloques completamente al azar, siendo el criterio la formación de lotes según el peso inicial, para evaluar la ganancia de peso, el consumo de alimento, conversión alimenticia y el rendimiento de carcasa, con un nivel de significancia de $\mathrm{p} \leq 0.05$. Los datos de rendimiento de carcasa que estaban en porcentaje fueron transformados a la proporción arco seno raíz cuadrada de la proporción para su análisis. Las diferencias entre medias se evaluaron con la prueba de Tukey; para la evaluación de animales retrasados y carcasas dañadas se evaluó con la prueba de Chi - Cuadrado. La retribución económica se determinó por diferencia entre el valor de unidad de carcasa ó kilogramo de carne y el costo de la alimentación.

\section{Resultados y discusión Ganancia de peso}

En el Tabla 2 se observan los pesos finales y ganancia de peso; con diferencias significativas $(\mathrm{p} \leq 0.05)$ a favor del 
tamaño de lote de 10 animales en comparación a los lotes de 30 y 50 animales que lograron resultados estadísticamente iguales. Estos resultados pueden deberse a que en el lote de 10 cuyes, los animales tuvieron menor exposición al estrés. $\mathrm{Al}$ haber menos animales en el lote, se maneja mejor la relación entre los animales: hay menos competencia, así como menos riñas y pérdida de energía como tal.

\section{Consumo de alimento}

El consumo del alimento total expresado en materia seca, no mostró diferencias estadísticas significativas entre todos los tratamientos. Es posible que esta uniformidad se haya debido a que todos tenían la misma área de crianza $\mathrm{y}$ de comederos, logrando que todos los animales tengan la misma oportunidad de consumir alimento; tal como mencionan Kornegay y Notter (1984) al indicar que las alteraciones en la ingesta de alimento está más influenciado por el espacio dado en el corral que por la cantidad de animales instalados en el mismo.

Tabla 2. Efecto del tamaño del lote en el comportamiento productivo de cuyes

\begin{tabular}{lccc}
\hline \multicolumn{1}{c}{ Parámetro } & T1 & T2 & T3 \\
\hline Área libre, $\mathrm{m}^{2} /$ animal & 0.0781 & 0.0781 & 0.0781 \\
Área Total, $\mathrm{m}^{2}$ tratamiento & 0.9 & 2.7 & 4.5 \\
$\mathrm{~N}^{\mathrm{o}}$ animales/poza & 10 & 30 & 50 \\
Peso & & & \\
Inicial, g & $267^{\mathrm{a}}$ & $268^{\mathrm{a}}$ & $269^{\mathrm{a}}$ \\
Final, g & $1049^{\mathrm{a}}$ & $993^{\mathrm{b}}$ & $998^{\mathrm{b}}$ \\
Ganancia de peso & & & \\
Total, g & $782^{\mathrm{a}}$ & $725^{\mathrm{b}}$ & $729^{\mathrm{b}}$ \\
Diario, g & $13.96^{\mathrm{a}}$ & $12.95^{\mathrm{b}}$ & $13.02^{\mathrm{b}}$ \\
Consumo en base seca & & & \\
Alimento balanceado, g & $2091^{\mathrm{a}}$ & $2061^{\mathrm{a}}$ & $2089^{\mathrm{a}}$ \\
Rastrojo de brócoli, g & 738 & 738 & 738 \\
Total, g & $2829^{\mathrm{a}}$ & $2798^{\mathrm{a}}$ & $2826^{\mathrm{a}}$ \\
Conversión alimenticia & $3.62^{\mathrm{a}}$ & $3.86^{\mathrm{b}}$ & $3.87^{\mathrm{b}}$ \\
Peso de carcasa, g & 744.9 & 696.2 & 701.49 \\
Rendimiento de carcasa & $71.01^{\mathrm{a}}$ & $70.11^{\mathrm{a}}$ & $70.29^{\mathrm{a}}$ \\
Animales & & & \\
$\mathrm{N}^{\mathrm{o}}$ Retrasados & $1^{\mathrm{a}}$ & $4^{\mathrm{a}}$ & $6^{\mathrm{a}}$ \\
$\mathrm{N}^{\mathrm{o}}$ Carcasas dañadas & $1^{\mathrm{a}}$ & $0^{\mathrm{a}}$ & $1^{\mathrm{a}}$ \\
\hline
\end{tabular}

$\left({ }^{\mathrm{a} b}\right)$ : letras diferentes indica que existe diferencia estadística $(\mathrm{p} \leq 0.05)$.

\section{Conversión alimenticia}

Se encontró diferencia estadística significativa entre los tratamientos $(\mathrm{p} \leq 0.05)$ siendo el lote de 10 animales mejor a los lotes de 30 y 50 animales (Tabla 1), pero los dos últimos valores fueron similares. Al tener los tratamientos el mismo consumo pero con diferentes ganancias de peso, se puede asumir que existe una pérdida de energía, derivada probablemente al estrés de convivir con mayor número de animales, así como las peleas o persecuciones entre ellos, por lo que cada animal no aprovechó de igual manera el valor nutritivo del alimento en cada tratamiento. De ello se deduciría que podría existir un efecto directo entre el tamaño de lote y el nivel de estrés y por ende mayor pérdida de energía que se manifiesta con menor ganancia de peso y peores conversiones alimenticias encontradas en los lotes de 30 y 50 animales.

\section{Animales retrasados en crecimiento}

Existe una relación directa manteniéndose la proporción de animales retrasados por cada diez animales en el lote de crianza, sin mostrar diferencia estadística entre los tratamientos (Tabla 1). Se evidenció que el retraso en el crecimiento se manifiesta mas notoriamente entre la $8 \mathrm{va}$ y 10ma semana de edad, lo que coincide con la pubertad y se presume que los más perjudicados son los cuyes cuyos pesos estarían por debajo del promedio al momento de formar los lotes. Estos resultados nos estarían indicando que al tener la crianza en un área adecuada, la uniformidad del lote inicial sería el punto crítico para asegurar un establecimiento de respeto entre ellos.

\section{Rendimiento de carcasa}

Los rendimientos de carcasa son presentados en el Tabla 1, no habiendo diferencia estadística significativa $(\mathrm{p} \leq 0.05)$ entre los diferentes tamaños de lotes. En ese sentido, el tamaño de lote no influyó en la capacidad de desarrollo corporal; lo que quiere decir que cada animal tuvo la misma capacidad de formar tejido corporal con el alimento consumido.

\section{Carcasa dañada}

El número de carcasas dañadas resultó en promedio un animal por tratamiento, independientemente del tamaño de lote. Este parámetro no mostró diferencia estadística entre tratamientos, observándose que algunos cuyes presentan un comportamiento agresivo (como individuo), por lo que se presentan carcasas dañadas sin relación al tamaño de lote. Si bien los animales comienzan a pelear desde la 8va semana de edad, ellos solo intentan morder para asustar y lograr así que respeten su espacio; sin que cause mayor daño en la carcasa; sin embargo, conforme avanza la madurez sexual se presenta mayor incidencia de enfrentamientos, registrándose finalmente algún animal del grupo más sumiso que termina con heridas en el cuerpo.

\section{Retribución económica}

Los resultados de la retribución económica se muestran en el Tabla 3. Al evaluar por unidad de carcasa resultó que la retribución económica es indiferente entre los tratamientos; de forma que si se comercializara como unidad de cuy, sea vivo o muerto, la retribución económica sea igualmente semejante entre los diversos tamaños de lote. Sin embargo, en la evaluación por kilogramo de carne se obtuvo el mayor beneficio económico con el lote de 10 animales. Es de esperar que, aunque no fue evaluado en el presente trabajo, el ahorro se dé por el menor costo en infraestructura al trabajar con mayores tamaños de lote. 
Tabla 3. Efecto del tamaño del lote sobre el costo de alimentación y la retribución económica

\begin{tabular}{|c|c|c|c|}
\hline Parámetro & T1 & T2 & T3 \\
\hline $\mathrm{N}^{\circ}$ animales/poza & 10 & 30 & 50 \\
\hline Peso vivo promedio cuy, (kg) & 1.049 & 0.993 & 0.998 \\
\hline Peso de carcasa, $(\mathrm{Kg})$ & 0.7449 & 0.6962 & 0.7015 \\
\hline \multicolumn{4}{|l|}{ a. Precio (S/.) } \\
\hline Por unidad de carcasa & 20.00 & 20.00 & 20.00 \\
\hline Por kg de carne & 25.00 & 25.00 & 25.00 \\
\hline \multicolumn{4}{|l|}{ b. Ingreso Bruto (S/.) } \\
\hline Por unidad de carcasa & 20.00 & 20.00 & 20.00 \\
\hline Por kg de carne & 18.63 & 17.41 & 17.54 \\
\hline \multicolumn{4}{|l|}{ Alimento } \\
\hline Consumo de concentrado $(\mathrm{kg})$ & 2.376 & 2.342 & 2.374 \\
\hline $\begin{array}{l}\text { Costo por consumo de concentrado } \\
(\mathrm{S} / .1 .2 / \mathrm{kg})\end{array}$ & 2.851 & 2.811 & 2.849 \\
\hline $\begin{array}{l}\text { Consumo de brocoli }(\mathrm{kg})(\mathrm{S} / .0 .06 / \\
\mathrm{kg})\end{array}$ & 6.146 & 6.146 & 6.146 \\
\hline Costo por consumo brocoli & 0.369 & 0.369 & 0.369 \\
\hline Costo Total de Alimentación (S/.) & 3.22 & 3.18 & 3.22 \\
\hline \multicolumn{4}{|l|}{ Retribución económica (S/.) } \\
\hline Por unidad de carcasa & 16.78 & 16.82 & 16.78 \\
\hline Por kg de carne & 15.41 & 14.23 & 14.32 \\
\hline \multicolumn{4}{|l|}{ Mérito económico (\%) } \\
\hline Por unidad de carcasa & 100.0 & 100.2 & 100.0 \\
\hline Por kg de carne & 100.0 & 92.34 & 92.93 \\
\hline
\end{tabular}

\section{Conclusiones}

Existe una relación directa entre el tamaño de lote y la respuesta productiva del animal, de manera que a lotes más pequeños, se obtiene los mejores resultados con diferencia estadística significativa para los parámetros ganancia de peso y conversión alimenticia; sin afectar por ello el consumo de materia seca, el número animales retrasados en su crecimiento, número de carcasas dañadas, ni el rendimiento de carcasa.

Los cuyes machos criados en lotes hasta la 8va semana de estudio (10ma semana de edad), no tienen problemas con respecto a las carcasas dañadas, ya que se observa que el problema se presenta en cualquier tamaño de lote y parece que la conducta agresiva es propia de cada individuo.

Con respecto a la retribución económica se puede concluir que al vender por unidad de carcasa es indiferente criar lotes 10, 30 y 50 animales por poza. En cambio al vender por kilogramo de carne conviene criar lotes pequeños; ya que existe mejor utilidad para el criador.

\section{Literatura citada}

Aguila, R. R. 1999. Estrés: El villano favorito. Acontecer Porcino. Año 1999 Vol. VII No 36.

Agustin, R.1973. Efecto del área y densidad de crianza en el engorde de cuyes (Tesis Ing. Zootecnista). Universida Nacional Agraria La Molina, Lima, Perú.

Carpenter, J. 1995. La complejidad del ambiente de un animal y los factores estresantes. Tecnología
Avipecuaria 8: 41 - 43.

Cayotopa, J. 1986. Rendimiento reproductivo y productivo en cuyes de acuerdo a la densidad por poza (Tesis Ing. Zootecnista). Universidad Nacional Pedro Ruiz Gallo., Lambayeque, Perú.

Humala, A. 1971. Efecto de tres áreas mínimas de corral por animal sobre la velocidad de crecimiento en cuyes. (Tesis Ing. Zootecnista). Universida Nacional Agraria La Molina, Lima, Perú.

Kornegay, E. and Notter, D. 1982. Effects of floor space and numbers of pigs per pen on performance. Pig News and Information 5 (2): 23 - 28

Montesinos, V. J. 1972. Efecto del número de animales por grupo en el engorde de cuyes. Lima-Perú.

Rivas, D. 1995. Prueba de crecimiento de cuyes (Cavia Porcellus) con restricción en el suministro de forraje. Universida Nacional Agraria La Molina, Lima, Perú.

Valverde, N. 2006. Evaluación de cuatro áreas de crianza por animal en el crecimiento de cuyes. TUniversida Nacional Agraria La Molina, Lima, Perú.

Wellock, I. J.; Emmans, G.C. and Kyriazakis, I. 2004. Modeling the effects of stressors on the performance of populations of pig. Journal of Animal Science 82(15): 2442 - 2450p. 